\title{
Use of penetrating keratoplasty in acute bacterial keratitis
}

\author{
JOHN C HILL \\ From the Department of Ophthalmology, Groote Schuur Hospital and University of Cape Town
}

SUMMARY Twenty-three patients with bacterial keratitis had penetrating keratoplasties performed for deep indolent ulceration or descemetoceles, during the acute period. The period in hospital (17.6 days) was significantly lower than for a control group (35.4 days) who were treated medically and had subsequent grafts. The number of grafts remaining clear was similar, $70 \%$ and $72 \%$ respectively. Eleven patients $(48 \%)$ of those who had an acute graft achieved corrected visual acuities of $6 / 12$ or better. No cases of reinfection occurred and no eyes were lost.

\begin{abstract}
With improved microsurgical techniques and instrumentation the success rate of corneal grafting has improved considerably. Despite this most surgeons are hesitant to operate on inflamed or infected eyes because of the danger of complications, including infection of the donor disc. However, on occasions an acute graft is vital to save the eyeball. Into this category fall corneal lacerations with loss of corneal tissue and ruptured descemetoceles unresponsive to medical therapy. Although not performed under ideal conditions we have found the outcome of these acute grafts to be satisfactory in many cases.

The medical treatment of indolent corneal ulcers and descemetoceles can be prolonged, requiring long periods of hospitalisation. The favourable outcome of corneal grafts in other acute conditions suggested that this could be a reasonable method of treatment for indolent ulcers and descemetoceles, especially if these disorders were central and likely to heal as heavily vascularised scars obscuring the visual axis, thus requiring a corneal graft at a later date.
\end{abstract}

\section{Patients and methods}

The management of patients admitted to this hospital with corneal ulceration follows a standard regimen. ${ }^{\prime}$ Corneal scrapings are taken for Gram stain and culture. Initial broad spectrum antimicrobial therapy is instituted and modified later according to the laboratory findings. If corneal thinning occurs, a

Correspondence to J C Hill, FRCS, Department of Ophthalmology, University of Cape Town Medical School, Observatory 7925, Cape Town, South Africa. topical anticollagenase (acetyl cysteine $10 \%$ ) is prescribed. Perforation is treated by fitting a soft bandage contact lens or by application of tissue adhesive. Failure to seal the defect is an indication for surgical intervention.

Patients with peripheral ulceration or descemetoceles, or those in whom the central ulceration was shallow and responded rapidly to medication, were treated as above. When descemetoceles or deep corneal ulcers were present centrally, the patients were offered a corneal graft as a primary procedure provided the following criteria were met. The lesion should be both small enough to be totally excisable, leaving a healthy rim of cornea, and be unresponsive, or slowly responsive, to therapy. All these lesions were such that, if treated medically, they were likely to heal leaving central corneal scars requiring corneal grafts at a later date. Twenty-five patients (17 men and eight women) received acute grafts, performed by the author, during the period August 1981 to April 1984. All had proved or presumed bacterial keratitis and 15 had descementoceles, three of which had perforated and not responded to medical therapy. The remaining 10 patients had central indolent corneal ulcers. Two patients from the descemetocele group failed to attend follow-up and were excluded from the study. A further group of 18 patients fulfilled the criteria for admission into the study but did not receive a graft initially. Either the patient refused operation or donor material was not available. This group (11 men and seven women) later received a graft and were used as a control group for our series. 
All corneal grafts were performed by a similar technique. Donor material was obtained from whole eyes preserved in a moist chamber at $4^{\circ} \mathrm{C}$ or from corneoscleral discs preserved in McCarey-Kaufman culture medium. ${ }^{2}$ Donor corneal discs were cut $0 \cdot 4$ $\mathrm{mm}$ larger than the recipient bed, and secured with a continuous $10 / 0$ nylon suture with a buried knot. No tissue matching was undertaken. Topical antibiotic therapy was continued and topical corticosteroids initiated postoperatively.

\section{Results}

Twenty-three patients received acute corneal grafts in this study; a further 18 patients with similar corneal disease, who had secondary grafts, are included as controls. The ages of the two groups were almost identical (Table 1) but there were relatively more females in the control group.

In the acute group 10 patients had indolent ulcers and 13 descemetoceles, two of which had perforated. Thirteen patients in the control group had indolent ulcers and five had descemetoceles.

The average period in hospital for the acute graft group was 17.6 days compared with 24.7 days for the control group, whose disease was treated medically. The subsequent admission for an average of $10 \cdot 7$ days for a secondary graft gave a total mean period in hospital of 35.4 days for the control group.

Twelve grafts in the acute group developed rejection episodes; these were reversed in six patients. From the control group eight patients had rejection episodes, but only three were reversible. Therefore $26 \%$ of the study group and $28 \%$ of the control group grafts were lost from rejection. Only one case of primary graft failure occurred, and this was in the study group. Considering all causes of graft failure, $70 \%$ of the acute grafts remained clear compared with $72 \%$ of the secondary control grafts. A number of patients in each group had a subsequent graft that was successful. These results are not included in this paper. The mean follow-up time was 20 and 23 months for the acute and control groups respectively.

A number of complications arose in the acute graft group (Table 2). Many of these problems were present at the time of operation and were related to the disease rather than the surgery. Apart from the six grafts that became opaque from irreversible rejection, a further graft developed marked stromal haze in the early postoperative period, attributed to primary graft failure. Two other grafts developed mild stromal haze following reversible rejection episodes.

Significant lens opacities developed in five eyes; in three these were present at operation and were associated with posterior synechiae and pupillary
Table 1 Clinical details and results of grafting

\begin{tabular}{|c|c|c|}
\hline & $\begin{array}{l}\text { Acute } \\
\text { graft group } \\
(n=23)\end{array}$ & $\begin{array}{l}\text { Secondary } \\
\text { graft group } \\
(n=18)\end{array}$ \\
\hline Sex M:F & $15: 8$ & $11: 7$ \\
\hline \multicolumn{3}{|l|}{ Age } \\
\hline Average & 44 & 48 \\
\hline Range & $18-64$ & $19-71$ \\
\hline Indolent ulcer & 10 & 13 \\
\hline \multicolumn{3}{|l|}{ Descemetocele } \\
\hline Intact & 11 & 5 \\
\hline Perforated & 2 & - \\
\hline \multicolumn{3}{|c|}{ Hospital admission (days) } \\
\hline Initial (range) & $17 \cdot 6(12-19)$ & $24 \cdot 7(11-35)$ \\
\hline Secondary & 0 & $10 \cdot 7(9-13)$ \\
\hline Total & $17 \cdot 6(12-19)$ & $35 \cdot 4(22-46)$ \\
\hline \multicolumn{3}{|l|}{ Rejection } \\
\hline Reversible & 6 & 3 \\
\hline Irreversible & $6(26 \%)$ & $5(28 \%)$ \\
\hline Total & $12(52 \%)$ & $8(44 \%)$ \\
\hline Clear grafts & $16(70 \%)$ & $13(72 \%)$ \\
\hline \multicolumn{3}{|c|}{ Follow-up (months) } \\
\hline Mean (range) & $20(8-34)$ & $23(12-40)$ \\
\hline
\end{tabular}

Table 2 Complications

Stromal haze

Cataract

Pupillary membrane

Posterior synechiae

Anterior synechiae

Glaucoma

Stepping

Resuture

Astigmatism $>4.5 \mathrm{D}$

Graft infection

membranes. One patient (Fig. 1) had a lens abscess and an extracapsular extraction was performed at the time of grafting. A further patient developed a cataract (Fig. 2) six months later, and an extracapsular extraction was then performed.

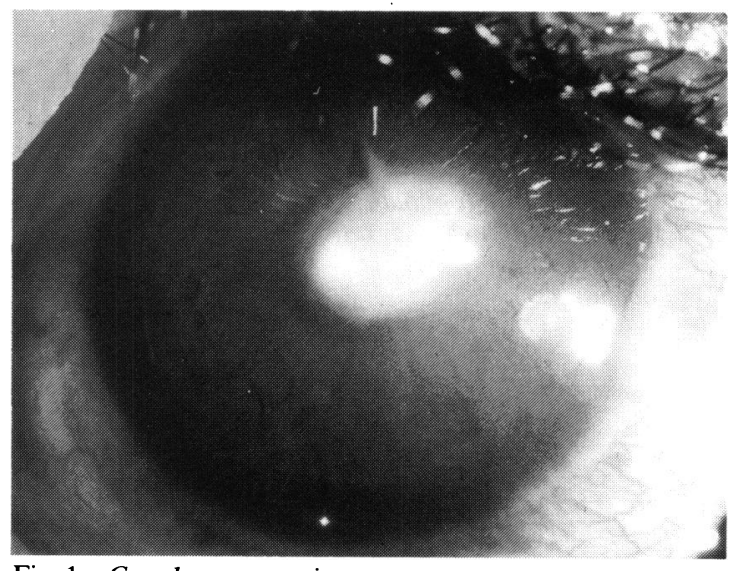

Fig. 1 Case 1, preoperative. 


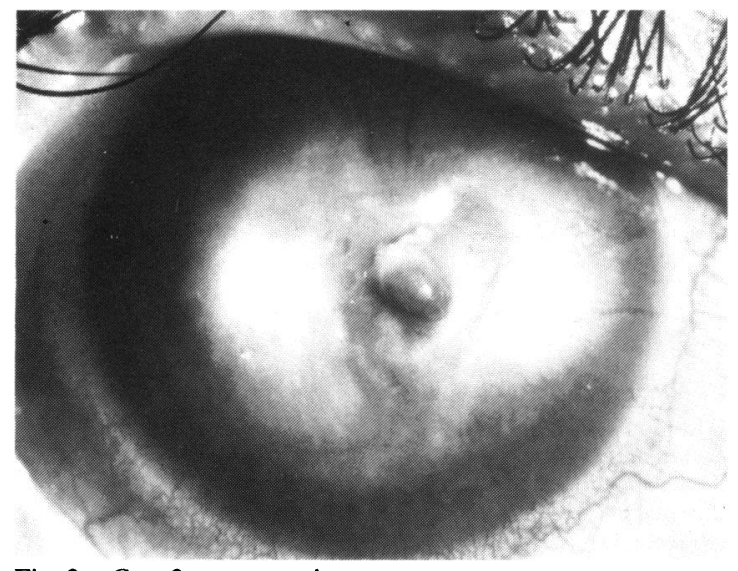

Fig. 2 Case 2, preoperative.

Inflammatory pupillary membranes were found in three patients. The membrane was left intact in one patient, but further synechiae developed causing pupil block glaucoma and iris bombé. A broad iridectomy with lens extraction was performed four weeks post graft. The other two pupillary membranes were removed at the time of grafting together with extracapsular lens extraction, because of anterior lens opacities. In addition to these three cases a further four patients developed posterior synechiae without sequelae.

Anterior synechiae developed in six patients. In two of them sodium hyaluronate was used to divide the synechiae in the early postoperative period. Three of these eyes developed glaucoma, and all were controlled with medical therapy. Glaucoma also occurred in the patient mentioned above with posterior synechiae and pupillary membrane.

In two patients stepping of the graft occurred owing to sutures 'cheese wiring.' In one resuturing was necessary. Both cases developed unacceptably high degrees of astigmatism, defined as a refractive astigmatism of greater than 4.5 dioptres. A further four patients developed excessive astigmatism; in two surgery had been performed on soft eyeballs with perforated descemetoceles. No cases of graft infection or endophthalmitis occurred.

In the 16 eyes that maintained clear grafts the final visual outcome varied (Fig. 3), because of complications such as minor lens opacities, stromal haze, or retinal problems. However, 11 (48\%) patients achieved corrected visual acuities of $6 / 12$ or better.

\section{CASE HISTORIES}

Case 1. A 36-year-old male injured his right eye with a piece of wood, sustaining a penetrating corneal wound. Two days later he had a central corneal abscess and a swollen infected lens (Fig. 1). Gram stain revealed scanty Gram-positive cocci but no growth was obtained. Topical and subconjunctival gentamicin and cephamandole were given, but the abscess slowly progressed. These two agents were then given systemically, whereupon the disease remained stationary. Sixteen days after admission a $7.5 \mathrm{~mm}$ graft was performed, a small hypopyon washed out, and an extracapsular lens extraction performed.

There were no postoperative complications and he left hospital nine days later. His graft has remained clear (Fig. 4) achieving a visual acuity of $6 / 7.5$ $\left(+13 \cdot 75 /+2 \cdot 25 \times 125^{\circ}\right)$, follow up 17 months.

Case 2. A 41-year-old woman gave a three-month history of a sore right eye that had been treated by her general practitioner. The cornea had a central abscess and descemetocele (Fig. 2). An $8.5 \mathrm{~mm}$ corneal graft was performed after two days of

\section{VISUAL OUTCOME}

Fig. 3 Visual outcome of patients receiving acute full-thickness grafts. $\mathrm{hm}=$ Hand movements. $\mathrm{cf}=$ Counting fingers. 


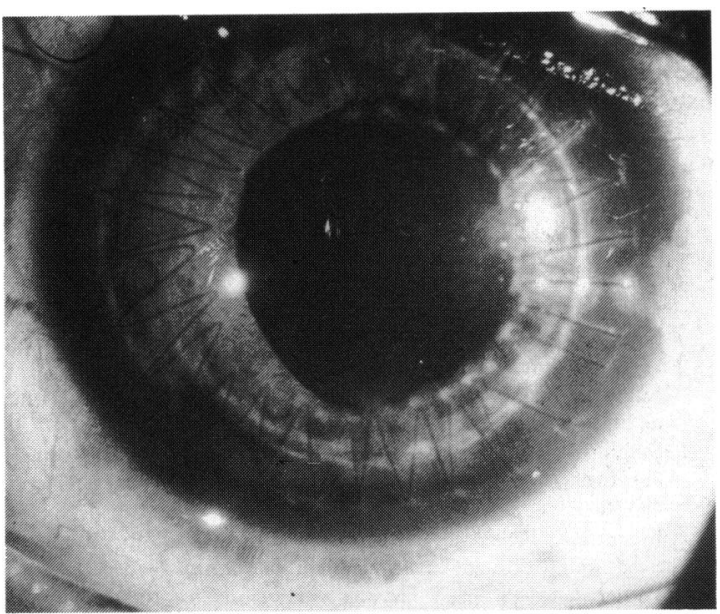

Fig. 4 Case 1, postoperative.

intensive topical gentamicin and cephamandole; no organisms were seen on Gram stain and there was no growth on culture. Recovery was uneventful, and she left the hospital nine days after admission. A small area of posterior synechiae formed, and she developed lens opacities (Fig. 5). An extracapsular lens extraction was performed six months post grafting. One month after lens extraction she developed a rejection response that was reversed, and her visual acuity remained at $6 / 12\left(+10 \cdot 50 /-3.00 \times 180^{\circ}\right)$ after a follow-up period of 21 months.

\section{Discussion}

Corneal ulcers can take a considerable time to heal despite being amenable to many forms of treatment that should ensure high tissue concentrations of therapeutic agents. Similarly patients with descemetoceles may need prolonged periods in hospital before the defect heals. In our control group, treated medically, an average of 24.7 days in hospital was required. After healing, a vascularised cornea often remains, which will increase the likelihood of rejection of a subsequent graft.

The surgical maxim recommending removal of dead or severely damaged tissue-for example, incising and draining an abscess-is as relevant to the cornea as elsewhere in the body. Even if the offending organism has been eradicated, various toxins and enzymes remain, resulting in further damage and the prevention of healing. Excision can be effected by keratoplasty. If the patient is likely to need a graft at some stage to restore vision, it is a reasonable proposition to perform this as soon as possible to allow early rehabilitation. Initially the diseased cornea is not vascularised. Therefore grafting at this stage may not be associated with a high incidence of

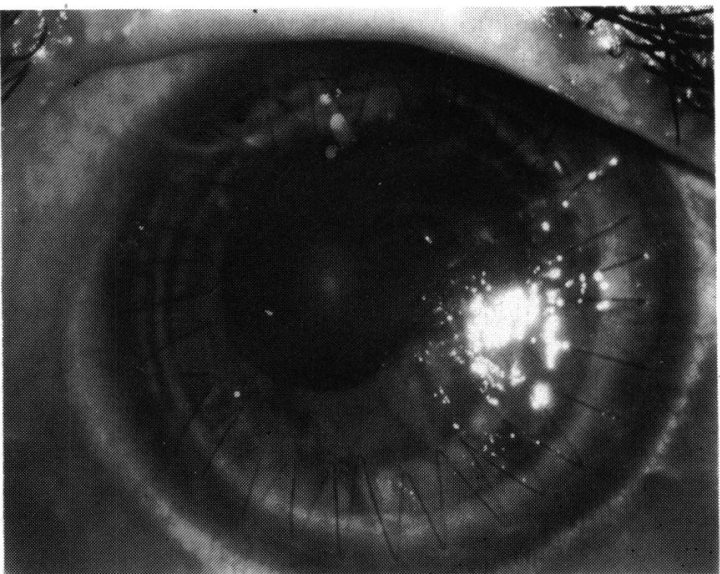

Fig. 5 Case 2, postoperative. Posterior synechiae and early anterior lens opacities are visible.

rejection. Although the eyes are inflamed, we have found the inflammation quickly subsides once the diseased cornea is excised.

Although keratoplasty has been used in fungal keratitis by a number of authors, ${ }^{3-6}$ this mode of treatment has not been widely reported in bacterial keratitis. Sporadic use of lamellar keratoplasty has been reported. ${ }^{7-11}$ Penetrating grafts have not been widely used in the past. ${ }^{10} 12$

Two more recent, larger series ${ }^{1314}$ have used both lamellar and penetrating keratoplasties in bacterial keratitis. We have been unhappy with the use of lamellar grafts in this condition because of the danger of reinfection. Malik and Singh ${ }^{13}$ reported recurrence of infection in all eight of their lamellar grafts, and one eye was lost. Du et al. ${ }^{14}$ used lamellar grafts if the infection was superficial. In our study lamellar grafts were not used; all corneas had deep stromal involvement. Patients with superficial disease were not offered surgery. We have found these infections to be less virulent and more responsive to medical therapy.

The average period in hospital for the acute graft group (17.6 days) was over a week less than for the control group (24.7 days). If we consider the subsequent admission for a secondary graft, the total mean period ( 35.4 days) becomes almost twice that for the acute graft group. Therefore early grafting decreases hospitalisation significantly and allows for early rehabilitation. $70 \%$ of the acute grafts remained clear compared with $72 \%$ for the control group and $67 \%$ in the penetrating keratoplasty group of Du et al. ${ }^{1+}(\mathrm{n}=38)$. It would appear that early surgery does not jeopardise the final outcome of the graft. The incidence of rejection was higher in the acute group (52\%) compared with the control group $(44 \%)$, though the failure rate from rejection is similar in the two groups, $26 \%$ and $28 \%$ respectively. 
Rejection was more difficult to reverse in the control group, and this may be related to the increased corneal vascularisation.

The number of complications is quite large, as would be expected in surgery of this type. However, as mentioned earlier, many of the complications are related and occurred in the same eye. The pupillary membranes were all associated with posterior synechiae, and all cases of glaucoma had anterior synechiae. One case of stepping required resuture and both developed excessive astigmatism. In this regard better results could have been obtained by operating before the descemetocele perforated. Unfortunately some patients present with leaking corneas and flat anterior chambers, and these require urgent surgery if they are unresponsive to medical therapy. There were two such cases in this series: both developed complications of anterior synechiae - glaucoma, unacceptable astigmatism, and in one a pupillary membrane with cataract. These patients, who required urgent surgery to salvage the eyeball, were operated upon for different criteria than the other patients. The equivalent in the control group does not of course exist, so their inclusion introduces a bias that makes comparison of complications inappropriate. If these two patients were excluded, the overall complication rate for the acute graft group drops considerably.

This study indicates that early grafting offers the advantage of shorter periods of hospitalisation and early rehabilitation. There may be an advantage in operating before vascularisation becomes established, but overall the final graft outcome does not differ significantly from that in a similar group of patients who were treated medically and had subsequent grafts to restore visual acuity.

\section{References}

1 Maske R, Hill JC, Oliver SP. The management of bacterial corneal ulcers. Br J Ophthalmol in press.

2 McCarey BE, Kaufman HE. Improved corneal storage. Invest Ophthalmol Vis Sci 1974; 13: 165-73.

3 Saunders N. Penetrating keratoplasty in treatment of fungus keratitis. Am J Ophthalmol 1970; 70: 24-30.

4 Singh G, Malik SRK. Therapeutic keratoplasty in fungal corneal ulcers. Br J Ophthalmol 1972; 56: 41-5.

5 Singh G, Malik SRK, Bhatnagar PK. Therapeutic value of keratoplasty in kerato mycosis. Arch Ophthalmol 1974; 92: 48-50.

6 Foster RK, Rebell G. Therapeutic surgery in failures of medical treatment for fungal keratitis. BrJ Ophthalmol 1975; 59: 366-71.

7 Paufique L, Philps S. Acute eye lesions treated by lamellar corneal grafting. Br J Ophthalmol 1950; 34: 746-8.

8 Paufique L. Indications for the therapeutic lamellar corneal graft. Am J Ophthalmol 1950; 33: 24-5.

9 Molnar L. Zur Behandelung des durch Pseudomonas pyocyanea verursachten Hornhautgeschwürs mit besonderer Berücksichtigung der therapeutischen Keratoplastik. Graefes Arch Clin Exp Ophthalmol 1960; 162: 1-7.

10 Ricci A. Greffe à chaud dans l'ulcère cornéen par pyocyanique. Ophthalmologica 1964; 147: 259-63.

11 Stern R, Lazar M. Our youngest case of a successful corneal graft. Ophthalmologica 1964; 148: 425-7.

12 Hallerman W. Unusual indications for keratoplasty. Surv Ophthalmol 1967; 12: 490-1.

13 Malik SRK, Singh G. Therapeutic keratoplasty in Pseudomonas pyocyaneas corneal ulcers. Br J Ophthalmol 1971; 55: 326-30.

14 Du NZ, Chen JQ, Gong XM, Xu HT, Feng CM. Therapeutic keratoplasty in the management of purulent corneal ulcerationreport of 100 cases. Jpn J Ophthalmol 1979; 23: 412-20.

Accepted for publication 14 November 1985. 